\title{
SOME TAMENESS CONDITIONS INVOLVING SINGULAR DISKS $\left({ }^{1}\right)$
}

\author{
BY \\ WARREN WHITE
}

Introduction. A familiar sort of lemma in the study of $E^{3}$ is the following:

Lemma. Let $D$ and $F$ be two disks in $E^{3}$ with $\partial D \cap F=D \cap \partial F=\varnothing$, and let $U$ be a neighborhood of $F^{\circ}$ in $E^{3}$. Then there is a disk $D^{\prime}$ in $E^{3}$ such that $\partial D^{\prime}=\partial D$, $D^{\prime} \subset D \cup U$, and $O\left(D^{\prime}, F\right) \subset U$ where $O\left(D^{\prime}, F\right)$ is $D^{\prime} \backslash F$ minus the component containing $\partial D$. $\left(D^{\prime} \backslash F\right.$ means $D^{\prime} \backslash D^{\prime} \cap F$. $)$

Theorem 4 generalizes this lemma, allowing $E$ to be a singular disk with its "interior" disjoint from its "boundary". It is necessary to redefine $O\left(D^{\prime}, E\right)$, and this is done in $\S 2$; the new definition is motivated by Lemma $5 \mathrm{~A}$.

Applications of Theorem 4 to the study of 2-spheres in $E^{3}$ are given in $\$ 6$. Burgess has shown (Theorem 7 in [6]) that a 2-sphere $S$ in $E^{3}$ is tame from the interior (i.e., $S \cup$ int $S$ is a 3-cell) if it is "locally spanned" by disks in the interior; Theorem 6 partially extends this result, letting the spanning disks be singular but imposing a condition on their boundaries. Corollary $6 \mathrm{~A}$ notes that $S$ is then tame from the interior if "small loops in $S$ can be shrunk to points in small subsets of the interior." Corollary 6B answers a question raised by Bing $[5, \S 5]$.

1. Notation and terminology. We use the letter $d$ to denote the Euclidean metric for Euclidean 3-space $E^{3}$, and let $\rho(f, g)=\sup _{x \in A} d(f(x), g(x))$ for any two maps $f$ and $g$ of a space $A$ into $E^{3}$. A map $f$ of a subspace of $E^{3}$ into $E^{3}$ is a $\delta$-map if $\rho(f, I)<\delta$, where $I$ is the identity map.

An $n$-manifold $N$ is a separable metric space such that each point $p \in N$ has an $n$-cell neighborhood in $N . N^{\circ}=\{p \in N: p$ has a neighborhood in $N$ homeomorphic to $\left.E^{n}\right\}$, and $\partial N=N \backslash N^{\circ} . N$ is an $n$-manifold-with-boundary if $\partial N \neq \varnothing$. A Euclidean neighborhood of a point $p \in N$ is an $n$-cell neighborhood $U$ together with a linear structure on $U$. If $S$ is a connected $(n-1)$-manifold in $N$ which separates $N$, and $V$ is a component of $N \backslash S$, then $S$ is tame from $V$ if $S \cup V$ is an $n$-manifold. All 2-manifolds and 3-manifolds are assumed to be triangulated [2, Theorem 6], and we use the same symbol for both the manifold and its triangulation.

Presented to the Society, January 23, 1968 under the title $A$ 2-sphere is tame if it is 1-LC through each complementary domain; received by the editors March 5, 1968.

$\left.{ }^{(}\right)$This paper is essentially the author's Ph.D. thesis written under Joseph Martin at the University of Wisconsin. The author was supported by a National Science Foundation Graduate Fellowship. 
Two subsets $X$ and $Y$ of an $n$-manifold $N$ are in relative general position if, for each point $p \in N$, there is a Euclidean neighborhood $U$ of $p$ and triangulations $T_{X}$ and $T_{Y}$ of $X \cap U$ and $Y \cap U$ such that

(i) each simplex of $T_{X}$ and $T_{Y}$ is a simplex in $U$,

(ii) dimension $\left(\left|T_{X}^{i}\right| \cap\left|T_{Y}^{j}\right|\right) \leqq i+j-n$.

A map $f: X \rightarrow N$ is in general position if, for each point $p \in N$, there is a Euclidean neighborhood $U$ of $p$ and a triangulation $T$ of $f^{-1}(U)$ such that

(i) for each simplex $\sigma \in T, f(\sigma)$ is a simplex in $U$,

(ii) for any two distinct simplices $\sigma_{1} \in T^{i}$ and $\sigma_{2} \in T^{j}$,

$$
\text { dimension }\left(f\left(\sigma_{1}^{\circ}\right) \cap f\left(\sigma_{2}^{\circ}\right)\right) \leqq i+j-n .
$$

If $X$ and $Y$ are two triangulated spaces, then $X \oplus Y$ denotes the disjoint union of both the spaces $X$ and $Y$ and their triangulations.

A Dehn disk $D$ in $E^{3}$ is the image of a real disk $\Delta$ under a map $f: \Delta \rightarrow E^{3}$ such that, for some subdisk $\Delta_{1} \subset \Delta^{\circ}, f\left(\Delta_{1}\right) \cap f\left(\Delta \mid \Delta_{1}\right)=\varnothing$ and $\left.f\right|_{\Delta \Delta_{1}}$ is piecewise linear and 1-1. The singularities of $f$ are the points of $\Delta$ in the closure of $\left\{x \in \Delta: f^{-1} f(x)\right.$ $\neq x\}$, and the singular points of $D$ are the images under $f$ of these singularities. $\partial D=f(\partial \Delta)$.

If $S$ is a 2 -sphere in $E^{3}$, then int $S$ and ext $S$ are, respectively, the bounded and unbounded components of $E^{3} \mid S$. Sierpinski curve and inaccessible point are as defined in [5].

2. Algebraic separation. Let $N$ be a simply-connected $n$-manifold, $n \leqq 3$. An (n-1)-polyhedron $K$ is an algebraic separator of $N$ if $K \cap N^{\circ}$ can be given a triangulation in which each $(n-2)$-simplex is the face of an even number of $(n-1)$-simplices.

Suppose that $K$ is an algebraic separator of $N$. Any arc $A \subset N$ in general position relative to $K$ hits $K$ at a finite number $\|A \cap K\|$ of points, and standard counting arguments show that:

Proposition 2A. If $A \subset N$ and $B \subset N$ are polygonal arcs in general position relative to $K$, and $A, B$ have the same endpoints, then $\|A \cap K\|=\|B \cap K\|(\bmod 2)$. In particular, if $\|A \cap K\|$ is odd then the endpoints of $A$ are separated in $N$ by $K$.

Suppose that $D$ is a disk and $K \subset D^{\circ}$ is an algebraic separator of $D$. It follows from Proposition 2A that we can define a map $\phi_{D \backslash K}$ on $D \backslash K$ by setting $\phi_{D \backslash K}(x)$ $=\|A \cap K\|(\bmod 2)$, where $A$ is any arc from $\partial D$ to $x$ in general position relative to $K$. We let $O(D, K)=\left\{x \in D \backslash K: \phi_{D \backslash K}(x)=1\right\}$.

Now suppose that $\Delta$ is a disk, $M$ a 3-manifold, and $f: \Delta \rightarrow M$ a map such that $\left.f\right|_{\Delta^{\circ}}$ is locally piecewise linear and in general position. Let $D \subset M$ be a polyhedral disk in general position relative to $f\left(\Delta^{\circ}\right)$, such that $\partial D \cap f(\Delta)=D \cap f(\partial \Delta)=\varnothing$.

Proposition 2B. $f^{-1}(D)=J_{1} \cup \cdots \cup J_{s}$, where the $J_{i}$ are disjoint simple closed curves. $f\left(J_{i}\right)$ and $f\left(\bigcup J_{i}\right)=D \cap f(\Delta)$ are algebraic separators of $D$, and $O\left(D, f\left(\cup J_{i}\right)\right)$ $\subset \bigcup O\left(D, f\left(J_{i}\right)\right)$. 
Proof. To check that $O\left(D, f\left(\bigcup J_{i}\right)\right) \subset \bigcup O\left(D, f\left(J_{i}\right)\right)$, just note that, for any polygonal arc $A$ in general position relative to $f\left(\bigcup J_{i}\right),\left\|A \cap f\left(\bigcup J_{i}\right)\right\|=\left\|A \cap f\left(J_{1}\right)\right\|$ $+\cdots+\left\|A \cap f\left(J_{s}\right)\right\|$. The other statements follow from the general position of $\left.f\right|_{\Delta^{\circ}}$, and of $D$ relative to $f\left(\Delta^{\circ}\right)$.

\section{Induction lemma.}

Lemma 3. Let $M$ be a 3-manifold-with-boundary, $D$ and $\Delta$ disks. Let $f: \Delta \rightarrow M$ be a simplicial map in general position, $U$ an open neighborhood of $f(\Delta)$ in $M$.

Suppose $i: D \rightarrow M$ is a simplicial embedding such that $i(D)$ is in general position relative to $f(\Delta), i(D) \cap \partial M=i(\partial D)$, and $i(\partial D) \cap U=i(D) \cap f(\partial \Delta)=\varnothing$.

If $O(i(D), f(J)) \notin U$ for some simple closed curve $J \subset f^{-1} i(D)$, then there is a polyhedral disk $D^{\prime}$ in $M$ such that:

(3.1) $\partial D^{\prime}=i(\partial D)$,

(3.2) $D^{\prime} \subset i(D) \cup U$,

(3.3) $(i(D) \backslash U) \backslash\left(D^{\prime} \backslash U\right) \neq \varnothing$.

Proof. Our proof will be analogous to those of Papakyriakopoulos [10] and Stallings [11], but where they dealt with maps of disks, we will be working with the map $i \oplus f: D \oplus \Delta \rightarrow M$ defined by $\left.i \oplus f\right|_{D}=i,\left.i \oplus f\right|_{\Delta}=f$. To measure the singularity of this map we use the complex $S(i \oplus f)$ defined by Stallings in his proof of [11, Lemma 3]; for completeness, we reproduce the definition here.

For any simplicial map $\gamma$ of a complex $X$ into a complex $Y$, a simplicial map $\gamma \times \gamma: X \times X \rightarrow Y \times Y$ can be constructed, where $X \times X$ and $Y \times Y$ are the cartesian products of complexes as defined in [7, p. 67]. We define $S(\gamma)$ to be the inverse image under $\gamma \times \gamma$ of the diagonal of $Y \times Y$; since this diagonal is a subcomplex of $Y \times Y$, it follows that $S(\gamma)$ is a subcomplex of $X \times X$. The useful property of $S(\gamma)$ is that, if $\Pi: Y \rightarrow Z$ is a simplicial map into some complex $Z$, then $S(\gamma) \subset S(\Pi \gamma)$, and $S(\gamma)=S(\Pi \gamma)$ if and only if $\Pi$ is $1-1$.

We will induct on the number $\mathscr{H}(i, f)$ of simplices in $S(i \oplus f)$; assume that $O(i(D), f(J)) \notin U$ for some simple closed curve $J \subset f^{-1} i(D)$.

To simplify notation, we will identify $D$ with $i(D)$ from this point on in the proof of Lemma 3.

Through standard combinatorial techniques and the ideas Stallings uses in proving Lemma 3 of [11], one can show:

Proposition 3A. There is a regular neighborhood $N$ of $D \cap f(\Delta)$ in $M$, a closed neighborhood $V$ of $D \cap f(\Delta)$ in $D$, and a piecewise linear embedding $h: D \times[-1,1]$ $\rightarrow N$ such that

(i) $h(x \times 0)=x$ and $h(x \times \pm 1) \in \partial N$ for all $x \in D \backslash V$,

(ii) $N \subset U \cup h((D \backslash V) \times[-1,1])$ and $f(\Delta) \cap h((D \backslash V) \times[-1,1])=\varnothing$,

(iii) the maps $i: D \rightarrow N$ and $f: \Delta \rightarrow N$ are simplicial.

The proof of the lemma splits into two parts, depending on whether or not $N$ is simply connected. 
Case I. $N$ simply connected:

Proposition 3B. The components $S_{1}, \ldots, S_{r}$ of $\partial N$ are spheres.

Proof. See 7.2 in [10].

Proposition 3C. If $p$ is a point of $O(D, f(J)) \backslash U$, then $h(p \times-1)$ and $h(p \times 1)$ lie in different spheres $S_{i}$.

Proof. If $E$ is the disk in $\Delta$ bounded by $J$, then it follows from the general position of $f$, and of $D$ relative to $f(\Delta)$, that $O(D, f(J)) \cup f(E)=K$ is an algebraic separator of $N . h(p \times[-1,1])$ is a polyhedral arc in general position relative to $K$ which hits $K$ once; by Proposition $2 \mathrm{~A}, h(p \times-1)$ and $h(p \times 1)$ are separated in $N$ by $K \subset N^{\circ}$, and must therefore lie in different components of $\partial N$.

Proposition 3D. If $p$ is a point of $O(D, f(J)) \backslash U$, then $\partial D$ bounds a polyhedral disk $D^{\prime}$ in $(D \backslash p) \cup U$. ( $D^{\prime}$ satisfies (3.1)-(3.3)).

Proof. Suppose $\partial D$ lies in $S_{1}$. By Proposition 3C, $S_{1}$ does not contain both $h(p \times-1)$ and $h(p \times 1)$. $\partial D$ misses $h(p \times \pm 1)$, so $\partial D$ bounds a disk $D_{1}$ in $S_{1}$ missing $h(p \times \pm 1)$. For any $x \in D \backslash V, D_{1}$ contains at most one of the two points $h(x \times-1)$ and $h(x \times 1)$, since $D$ is an algebraic separator of $N$ separating them. Using the embedding of $D \times[-1,1]$ in $N$ given by Proposition $3 \mathrm{~A}$, we can therefore draw $D_{1}$ homeomorphically into $(D \backslash p) \cup U$.

Case II. $N$ not simply connected:

Let $\left(N_{1}, p\right)$ be the universal (simply connected) covering space for $N . N_{1}$ is a 3-manifold-with-boundary, and we triangulate $N_{1}$ so that $p: N_{1} \rightarrow N$ is simplicial. Let $U_{1}=p^{-1}(U \cap N)$.

Let $f_{1}: \Delta \rightarrow N_{1}$ be a lifting of $f$, and let $i_{1}, i_{2}, \ldots, i_{k}, \ldots: D \rightarrow N_{1}$ be the distinct liftings of $i$.

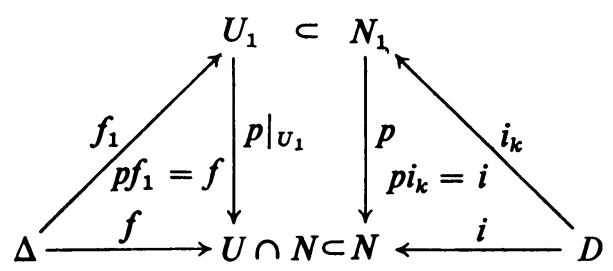

It is easy to check that

Proposition 3E. The hypotheses of Lemma 3 are satisfied by the substitution:

$\begin{array}{cccc}\text { for } & \text { substitute } & \text { for } & \text { substitute } \\ M & N_{1} & U & U_{1} \\ \Delta & \Delta & D & D \\ f & f_{1} & i & \text { any } i_{k}\end{array}$


Proposition 3F. For any $i_{k}, \mathscr{H}\left(i_{k}, f_{1}\right)<\mathscr{H}(i, f)$.

Proof. Consider the commutative diagram:

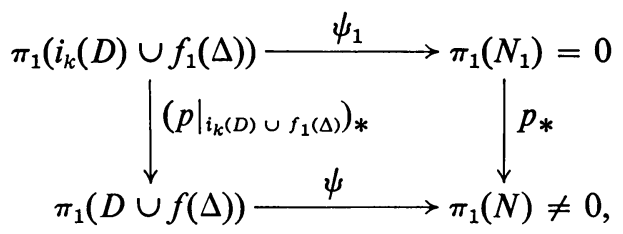

where $\psi_{1}$ and $\psi$ are induced by inclusions. $\psi$ is onto since $N$ is a regular neighborhood of $D \cup f(\Delta)$.

Now, $\quad S\left(i_{k} \oplus f_{1}\right) \subset S\left(p \circ\left(i_{k} \oplus f_{1}\right)\right)=S(i \oplus f)$. If $\quad S\left(i_{k} \oplus f_{1}\right)=S(i \oplus f)$, then $p \mid i_{k}(D) \cup f_{1}(\Delta)$ is $1-1$ and hence a homeomorphism, so $\left(p \mid i_{k}(D) \cup f_{1}(\Delta)\right)_{*}$ is onto. But then

$$
0 \neq \pi_{1}(N)=\psi\left(\left.p\right|_{i_{k}(D) \cup f_{1}(\Delta)}\right)_{*} \pi_{1}\left(i_{k}(D) \cup f_{1}(\Delta)\right)=p_{*} \psi_{1} \pi_{1}\left(i_{k}(D) \cup f_{1}(\Delta)\right)=0,
$$

a contradiction. Thus, $S\left(i_{k} \oplus f_{1}\right)$ is properly contained in $S(i \oplus f)$, and $\mathscr{H}\left(i_{k}, f_{1}\right)$ $<\mathscr{H}(i, f)$.

Proposition 3G. For some $K, J \subset f_{1}^{-1} i_{K}(D)$ and $O\left(i_{K}(D), f_{1}(J)\right) \notin U_{1}$.

Proof. $J \subset f^{-1}(D)=\left(p f_{1}\right)^{-1}(D)=f_{1}^{-1}\left(p^{-1}(D)\right)=f_{1}^{-1}\left(\bigcup i_{k}(D)\right)=\bigcup f_{1}^{-1} i_{k}(D)$; since the disks $i_{k}(D)$ are disjoint, $J \subset f_{1}^{-1} i_{K}(D)$ for some $K$. $p i_{K}=i$ is a homeomorphism, so $p\left(O\left(i_{K}(D), f_{1}(J)\right)=O(D, f(J))\right.$; if $O\left(i_{K}(D), f_{1}(J)\right) \subset U_{1}$, then $O(D, f(J)) \subset p\left(U_{1}\right)$ $=U \cap N$, a contradiction to our assumption that $O(D, f(J)) \notin U$.

Proposition 3H. There is a polyhedral disk $D^{\prime}$ in $M$ satisfying (3.1)-(3.3).

Proof. Let $D_{1}=i_{K}(D)$, where $K$ is given by Proposition 3G. By our induction, there is a polyhedral disk $D_{1}^{\prime}$ in $N_{1}$ such that:

(i) $\partial D_{1}^{\prime}=\partial D_{1}$,

(ii) $D_{1}^{\prime} \subset D_{1} \cup U_{1}$,

(iii) $\left(D_{1} \mid U_{1}\right) \mid\left(D_{1}^{\prime} \backslash U_{1}\right) \neq \varnothing$.

Since $\left.p\right|_{D_{1}}$ is a homeomorphism, the singularities of $p: D_{1}^{\prime} \rightarrow M$ all lie in $U_{1}$. $p\left(U_{1}\right) \cap p\left(\partial D_{1}^{\prime}\right) \subset U \cap \partial D=\varnothing$, so we can apply Dehn's lemma [9, Theorem IV.3] to get a polyhedral disk $D^{\prime}$ in $M$ such that:

(iv) $\partial D^{\prime}=p\left(\partial D_{1}^{\prime}\right)$

(v) $D^{\prime} \subset p\left(D_{1}^{\prime}\right) \cup U$.

It is easy to check that (i)-(v) imply that $D^{\prime}$ satisfies (3.1)-(3.3).

\section{Using a singular disk to "cut back" a real disk.}

THEOREM 4. Let $U_{0}$ be an open subset of $E^{3}, \Delta_{0}$ a disk, and $f_{0}: \Delta_{0} \rightarrow E^{3}$ a map such that $f_{0}\left(\Delta_{0}\right) \cap U_{0}=f_{0}\left(\Delta^{\circ}{ }_{0}\right)$ and $\left.f_{0}\right|_{\Delta_{0}^{\circ}}: \Delta^{\circ}{ }_{0} \rightarrow E^{3}$ is locally piecewise linear and in general position. 
Suppose that $D \subset E^{3}$ is a polyhedral disk such that $D \cap f_{0}\left(\partial \Delta_{0}\right)=\partial D \cap f_{0}\left(\Delta_{0}\right)=\varnothing$. Then there is a polyhedral disk $D^{\prime}$ in $E^{3}$ such that

(4.1) $\partial D^{\prime}=\partial D$,

(4.2) $D^{\prime} \subset D \cup U_{0}$,

(4.3) $D^{\prime}$ is in general position relative to $f_{0}\left(\Delta_{0}^{\circ}\right)$,

(4.4) $O\left(D^{\prime}, D^{\prime} \cap f_{0}\left(\Delta_{0}\right)\right) \subset U_{0}$.

Proof. We may assume that $\bar{U}_{0}$ is locally polyhedral $\bmod f_{0}\left(\partial \Delta_{0}\right)$, and that $U_{0} \cap \partial D=\varnothing$. For any disk $D^{\prime}$ satisfying (4.1) and (4.2), let $\mathscr{H}\left(D^{\prime}\right)$ be the number of components of $D^{\prime} \backslash U_{0} ; \mathscr{H}\left(D^{\prime}\right)$ is finite because $\bar{U}_{0}$ is polyhedral near $D^{\prime}$.

$D$ satisfies (4.1) and (4.2); we will induct on $\mathscr{H}(D)$. By adjusting $D$ within $U_{0}$, if necessary, we may assume that $D$ is in general position relative to $f_{0}\left(\Delta_{0}^{\circ}\right)$; if $O\left(D, D \cap f_{0}\left(\Delta_{0}\right)\right) \subset U_{0}$, as is the case when $\mathscr{H}(D)=1$, then we have nothing to prove. Suppose that $O\left(D, D \cap f_{0}\left(\Delta_{0}\right)\right) \notin U_{0}$.

Since $\left(D \cup U_{0}\right) \cap f_{0}\left(\partial \Delta_{0}\right)=\varnothing$, we can choose a disk $\Delta \subset \Delta_{0}^{\circ}$ such that $f=$ $\left.f_{0}\right|_{\Delta}: \Delta \rightarrow E^{3}$ is piecewise linear and in general position, and

$$
D \cap f_{0}\left(\Delta_{0}\right) \subset f\left(\Delta^{\circ}\right) \backslash f(\partial \Delta) .
$$

Using standard Euclidean-space techniques, together with the fact that

one can show:

$$
f_{0}\left(\Delta_{0}^{\circ}\right) \cap f_{0}\left(\partial \Delta_{0}\right)=\varnothing
$$

Proposition 4A. There is a 3-manifold-with-boundary $M \subset E^{3}$ such that

(i) $D \cup f(\Delta) \subset M$,

(ii) $D \cap \partial M=\partial D$,

(iii) $M \cap f_{0}\left(\partial \Delta_{0}\right)=\varnothing$.

Furthermore, $M, D$, and $\Delta$ may be triangulated so that the hypotheses of Lemma 3 are satisfied by $M, D, \Delta, f, U=U_{0} \cap M$, and the natural injection $i: D \rightarrow M$.

Since $O\left(D, D \cap f_{0}\left(\Delta_{0}\right)\right) \notin U_{0}$, we have also $O(D, D \cap f(\Delta)) \notin U$. By Proposition 2B, $O(D, D \cap f(J)) \notin U$ for some simple closed curve $J \subset f^{-1}(D)$. Lemma 3 then gives us a polyhedral disk $D^{\prime}$ such that

(4.1) $\partial D^{\prime}=\partial D$,

(4.2) $D^{\prime} \subset D \cup U \subset D \cup U_{0}$,

(3.3) $(D \backslash U) \mid\left(D^{\prime} \backslash U\right) \neq \varnothing$.

To show that (3.3) implies $\mathscr{H}\left(D^{\prime}\right)<\mathscr{H}(D)$, we note

Proposition 4B. $D^{\prime} \backslash U=D^{\prime} \backslash U_{0}, D \backslash U=D \backslash U_{0}$, and each component of $D^{\prime} \backslash U$ is a component of $D \mid U$.

Proof. $D^{*}\left|U=D^{*}\right|\left(U_{0} \cap M\right)=\left(D^{*} \mid U_{0}\right) \cup\left(D^{*} \mid M\right)=D^{*} \mid U_{0}$, where $D^{*}$ is either $D^{\prime}$ or $D$. That the components of $D^{\prime} \backslash U$ are components of $D \backslash U$ follows from (4.1) and (4.2) above.

REMARK. The proof of Theorem 4 shows that we can actually have $D^{\prime}$ satisfy (4.4') $O\left(D^{\prime}, D^{\prime} \cap f_{0}(J)\right) \subset U_{0}$, for each simple closed curve $J \subset f_{0}^{-1}\left(D^{\prime}\right)$. 
5. Applying Theorem 4. Throughout the remainder of the paper, $\Delta$ will represent a standard disk. Let $M$ be a 3-manifold, $S$ a 2-manifold in $M$, and $F \subset S$ a disk.

Proposition 5A. Let $\mathscr{G}$ be the class of all maps $g: \partial \Delta \rightarrow F^{\circ}$ which are piecewise linear into $F$ and in general position. Then, for an arbitrary map $f: \partial \Delta \rightarrow F$, $\phi_{F \backslash f(\partial \Delta)}=\lim _{g \in \mathscr{G} ; \rho(f, g) \rightarrow 0} \phi_{F \mid g(\partial \Delta)}$ exists on $F \backslash f(\partial \Delta)$, and $\phi_{F \backslash f(\partial \Delta)}(x)=\phi_{F \mid g(\partial \Delta)}(x)$ for any map $g \in \mathscr{G}$ which is homotopic to $f$ in $F \backslash x$.

Proof. Both assertions follow from the easily demonstrated fact that if two maps $g_{1}$ and $g_{2}: \partial \Delta \rightarrow F$ are piecewise linear into $F$, in general position, and homotopic in $F \mid x$, then $\phi_{F \mid g_{1}(\partial \Delta)}(x)=\phi_{F \mid g_{2}(\partial \Delta)}(x)$.

If $V$ is a component of $M \backslash S$, then a blister of $F$ in $V$ is a map $f: \Delta \rightarrow F \cup V$ such that $f(\Delta) \cap S=f(\partial \Delta)$. We let $O(F, f)=\left\{x \in F \backslash f(\partial \Delta): \phi_{F \backslash f(\partial \Delta)}(x)=1\right\}$, and denote $f(\Delta) \cup O(F, f)$ by $(f)_{F}$.

Lemma 5A. Let $S$ be a 2-sphere in $E^{3}, F \subset S$ a disk. Let $f$ be a blister of $F$ in int $S$, and $B$ a 3-cell in $E^{3}$, such that $(f)_{F} \subset B^{\circ}$ and $\left.f\right|_{\Delta^{\circ}}: \Delta^{\circ} \rightarrow E^{3}$ is locally piecewise linear and in general position.

Suppose $p$ is a point of $O(F, f), q$ is a point of int $S \backslash B$, and $q p \subset E^{3}$ is a polygonal arc in general position relative to $f\left(\Delta^{\circ}\right)$ such that $q p \backslash p \subset$ int $S$. Then $\|q p \cap f(\Delta)\|$ is odd.

Proof. Pick a point $r$ in ext $S \backslash B$. We can use Theorem 5.37 of [12] to extend $q p$ to a polygonal arc $q p r \subset E^{3}$ such that $p r$ misses $S \backslash O(F, f)$ and $f(\Delta)$.

Let $\delta=d\left(f(\partial \Delta),\left(E^{3} \mid B\right) \cup q p r\right)$, and triangulate $S$ so that $F$ is a polyhedron in $S$. Using Bing's approximation theorem [1, Theorem 1], we can find a piecewise linear $\delta / 2$-homeomorphism $h: S \rightarrow E^{3}$ such that

(1) $q p r \cap h(S) \subset h(O(F, f))$

$$
\left(=\left\{x \in h(F) \mid h f(\partial \Delta): \phi_{h(F) \mid h f(\partial \Delta)}(x)=1\right\}\right),
$$

(2) $q \in$ int $h(S), r \in \operatorname{ext} h(S)$,

(3) $h(S)$ is in general position relative to $f\left(\Delta^{\circ}\right) \cup q p r$.

Proposition 5B. There is a polyhedron $K \subset B \subset E^{3}$ such that:

(i) $K$ is an algebraic separator of $E^{3}$ in general position relative to qpr.

(ii) $q p r \cap(f(\Delta) \cup h(S))=q p r \cap K$.

Proof. Let $g: \partial \Delta \rightarrow E^{3}$ be a piecewise linear map in general position, such that:

(i) $g(\partial \Delta) \subset h\left(F^{\circ}\right)$,

(ii) $g$ and $\left.h f\right|_{\partial \Delta}$ are homotopic in $h(F) \mid q p r$,

(iii) $\rho\left(g,\left.h f\right|_{\partial \Delta}\right)<\delta / 2$.

Let $\gamma: \Delta \rightarrow \Delta^{\circ}$ be a homeomorphism such that

(iv) $\rho(f \gamma, f)<\delta$,

(v) $f \gamma: \Delta \rightarrow E^{3}$ is piecewise linear and in general position, and $f \gamma(\Delta)$ is in general position relative to $h(S)$. 
As a result of our care with $\delta$, we can get a piecewise linear homotopy $G: \partial \Delta \times[0,1] \rightarrow E^{3}$ such that

(vi) $G_{0}=g, G_{1}=\left.f \gamma\right|_{\partial \Delta}$,

(vii) $G(\partial \Delta \times[0,1]) \subset B \mid q p r$,

(viii) $G(\partial \Delta \times(0,1))$ is in general position relative to $h(S)$ and $f \gamma(\Delta)$.

It is simple to check that $K=O(h(F), g(\partial \Delta)) \cup G(\partial \Delta \times[0,1]) \cup f \gamma(\Delta)$ satisfies the requirements.

Proposition 5C. $\|q p \cap f(\Delta)\|$ is odd.

Proof. $K$ is contained in $B$, which does not separate $q$ and $r$ in $E^{3}$, so by Proposition 2A $\|q p r \cap K\|$ is even. $\|q p r \cap K\|=\|q p r \cap(f(\Delta) \cup h(S))\|=\|q p r \cap f(\Delta)\|$ $+\|q p r \cap h(S)\|$, by condition (3) on $h$. $\|q p r \cap h(S)\|$ is odd since $h(S)$ is a manifold separating $q$ and $r$ in $E^{3}$, so $\|q p r \cap f(\Delta)\|$ is also odd.

LEMMA 5B. Let $S$ be a 2-sphere in $E^{3}, F \subset S$ a disk. Let $f_{1}, \ldots, f_{s}$ be blisters of $F$ in int $S$, and $B_{1}, \ldots, B_{s}$ 3-cells in $E^{3}$ such that $\left(f_{i}\right)_{F} \subset B_{i}^{\circ}$ for each $i$.

Suppose $D$ is a polyhedral disk in $E^{3}$ such that $\partial D \subset$ int $S \backslash \cup B_{i}$, int $S \cup$ $\left(F \backslash \bigcup f_{i}(\partial \Delta)\right) \cup D$ retracts to int $S \cup\left(F \backslash \cup f_{i}(\partial \Delta)\right)$, and $D \cap S \subset \cup O\left(F, f_{i}\right)$.

Then there is a disk $D^{\prime}$ in $E^{3}$ such that

(5.1) $\partial D^{\prime}=\partial D$,

(5.2) $D^{\prime} \subset D \cup\left(\cup B_{i}\right)$,

(5.3) $D^{\prime} \subset$ int $S$.

Proof. Suppose that we have a polyhedral disk $D_{j}$ in $E^{3}$ which satisfies the following conditions:

(1) $\partial D_{j}=\partial D$,

(2) $D_{j} \subset D \cup\left(\cup B_{i}\right)$,

(3) int $S \cup\left(F \backslash \cup f_{i}(\partial \Delta)\right) \cup D_{j}$ retracts to int $S \cup\left(F \backslash \cup f_{i}(\partial \Delta)\right)$,

(4) $D_{j} \cap S \subset \bigcup_{j<i \leqq s} O\left(F, f_{i}\right)$.

We can choose $D_{0}=D$, for example, and if we had $D_{s}$ we could choose $D^{\prime}=D_{s}$. For the proof of Lemma $5 \mathrm{~B}$ it is, therefore, sufficient to produce $D_{j+1}$.

We may assume that, for each $i,\left.f_{i}\right|_{\Delta^{\circ}}$ is locally piecewise linear and in general position. The hypotheses of Theorem 4 are then satisfied by the following substitutions:

$\begin{array}{cccc}\text { for } & \text { substitute } & \text { for } & \text { substitute } \\ \Delta_{0} & \Delta & U_{0} & B_{j+1} \cap \text { int } S \\ f_{0} & f_{j+1} & D & D_{j}\end{array}$

There is, therefore, a polyhedral disk, which we shall call $D_{j+1}$, satisfying:

(4.1) $\partial D_{j+1}=\partial D_{j}$,

(4.2) $D_{j+1} \subset D_{j} \cup\left(B_{j+1} \cap\right.$ int $\left.S\right)$,

(4.3) $D_{j+1}$ is in general position relative to $f_{j+1}\left(\Delta^{\circ}\right)$,

(4.4) $O\left(D_{j+1}, D_{j+1} \cap f_{j+1}(\Delta)\right) \subset B_{j+1} \cap$ int $S$. 
From (4.1) and (4.2) it follows that $D_{j+1}$ satisfies conditions (1)-(3); it remains to check (4).

Proposition 5D. $D_{j+1} \cap S \subset \bigcup_{j+1<i \leqq s} O\left(F, f_{i}\right)$.

Proof. (4.2) implies that $D_{j+1} \cap S \subset \bigcup_{j<i \leqq s} O\left(F, f_{i}\right)$, so all we need check is $O\left(F, f_{j+1}\right)$. Suppose that $D_{j+1} \cap O\left(F, f_{j+1}\right) \neq \varnothing$, and use (4.3) to choose a polygonal arc $A$ with endpoints $p$ and $q$, such that

(i) $A \subset D_{j+1}$,

(ii) $q \in \partial D_{j+1}, p \in O\left(F, f_{j+1}\right)$,

(iii) $A$ is in general position relative to $f_{j+1}\left(\Delta^{\circ}\right)$.

Using the facts that $D_{j+1}$ satisfies (3) and int $S$ is locally 0 -connected [12, Theorem 5.35], we can get a polygonal arc $A^{\prime}$ with endpoints $p^{\prime}$ and $q$, such that

(iv) $A^{\prime} \backslash p^{\prime} \subset$ int $S$,

(v) $p^{\prime} \in O\left(F, f_{j+1}\right)$

(vi) $A^{\prime} \cap W=A \cap W$, for some neighborhood $W$ of $f_{j+1}\left(\Delta^{\circ}\right)$ in int $S$.

$A^{\prime}$ is in general position relative to $f_{j+1}\left(\Delta^{\circ}\right)$ since $A$ is, so Lemma $5 \mathrm{~A}$ tells us that $\left\|A^{\prime} \cap f_{j+1}(\Delta)\right\|$ is odd. Since $\left\|A^{\prime} \cap f_{j+1}(\Delta)\right\|=\left\|A \cap f_{j+1}(\Delta)\right\|$, this means that $p \in O\left(D_{j+1}, D_{j+1} \cap f_{j+1}(\Delta)\right)$. According to (4.4), $p$ then lies in int $S$; but we assumed that $p \in S$, which is a contradiction. Therefore, $D_{j+1} \cap O\left(F, f_{j+1}\right)=\varnothing$.

6. 2-spheres in $E^{3}$. Let $M$ be a 3-manifold, $S$ a 2-manifold in $M$, and $V$ a component of $M \backslash S$. S satisfies condition (1) toward $V$ at a point $p \in S$ if, for any neighborhood $B$ of $p$ in $M$, and any Cantor set $C$ in $S$, there is a disk $F \subset S \cap B$ and a blister $f$ of $F$ in $V$ such that $p \in O(F, f) \subset(f)_{F} \subset B$ and $f(\partial \Delta) \cap C=\varnothing$.

THEOREM 6. Let $S$ be a 2-sphere in $E^{3}$ which satisfies condition (1) toward its interior at every point. Then $S$ is tame from the interior.

Proof. Let $F \subset S$ be a disk, $U$ a neighborhood of $F$ in $E^{3}$, and $D \subset E^{3}$ a polyhedral disk with $\partial D \subset$ int $S$ and $D \cap S \subset F^{\circ}$.

Proposition 6A. To prove Theorem 6, it is sufficient to show that there is a disk $D^{\prime}$ in $E^{3}$ such that

(i) $\partial D^{\prime}=\partial D$,

(ii) $D^{\prime} \subset D \cup U$,

(iii) $D^{\prime} \subset$ int $S$.

Proof. As Hempel has noted (in the proof of [8, Theorem 1]), this is a consequence of Bing's proof of Theorem 1 in [3].

Proposition 6B. There is a Dehn disk $D_{0}$ in $E^{3}$ and a Cantor set $C$ in $S$ such that

(i) $\partial D_{0}=\partial D$,

(ii) $D_{0} \subset D \cup U$, with the singular points of $D_{0}$ contained in $U$,

(iii) $D_{0} \subset$ int $S \cap\left(F^{\circ} \cap C\right)$. 
Proof. We can use the Tietze extension theorem to get a Dehn disk $D_{0}^{\prime}$ such that $\partial D_{0}^{\prime}=\partial D$ and $D_{0}^{\prime} \subset(D \cap$ int $S) \cup F^{\circ}$, with the singular points of $D_{0}^{\prime}$ contained in $F$. Theorem 2.1 of [5] then gives us $D_{0}$.

Proposition 6C. There are blisters $f_{1}, \ldots, f_{s}$ of $F$ in int $S, 3$-cells $B_{1}, \ldots, B_{s}$ in $U \backslash \partial D$, and disjoint disks $G_{1}, \ldots, G_{r}$ in $S$, such that $\left(f_{i}\right)_{F} \subset B_{i}^{\circ}$ for each $i$, and $D_{0} \cap S \subset \bigcup G_{j}^{\circ} \subset \bigcup G_{j} \subset\left(\bigcup O\left(F, f_{i}\right)\right) \backslash \bigcup f_{i}(\partial \Delta)$.

Proof. For any point $p$ in $F^{\circ}$, we can choose a 3-cell neighborhood $B$ in $U \backslash(\partial D \cup(S \backslash F))$. Let $C$ be the Cantor set described in Proposition 6B; since $S$ satisfies condition (1) toward int $S$ at $p$, there is a disk $F_{p} \subset S \cap B_{p} \subset F$ and a blister $f_{p}$ of $F_{p}$ in int $S$ such that $p \in O\left(F_{p}, f_{p}\right)=O\left(F, f_{p}\right) \subset\left(f_{p}\right)_{F} \subset B_{p}^{\circ}$ and $f_{p}(\partial \Delta)$ $\cap C=\varnothing . D_{0} \cap S$ is compact, so we can pick $p_{1}, \ldots, p_{s} \in F^{\circ}$ such that $D_{0} \cap S$ $\subset\left(\bigcup O\left(F, f_{p_{i}}\right)\right) \backslash \bigcup f_{p_{i}}(\partial \Delta)$. We let $\left\{f_{1}, \ldots, f_{s}\right\}=\left\{f_{p_{1}}, \ldots, f_{p_{s}}\right\},\left\{B_{1}, \ldots, B_{s}\right\}=\left\{B_{p_{1}}, \ldots, B_{p_{s}}\right\}$, and use the fact that $D_{0} \cap S \subset C$ is 0 -dimensional to choose the disks $G_{1}, \ldots, G_{r}$.

We can use the Tietze extension theorem to show:

Proposition 6D. int $S \cup\left(\cup G_{j}\right)$ is a retract of some neighborhood $V$ of int $S$ $\cup\left(\bigcup G_{j}^{\circ}\right)$ in $E^{3}$.

Proposition 6E. There is a polyhedral disk $D_{1}$ in $E^{3}$ such that

(i) $\partial D_{1}=\partial D$

(ii) $D_{1} \subset D \cup U$,

(iii) $S, F,\left\{f_{1}, \ldots, f_{s}\right\},\left\{B_{1}, \ldots, B_{s}\right\}$, and $D=D_{1}$ satisfy the hypotheses of Lemma $5 B$.

Proof. The singular points of $D_{0}$ are contained in $U \cap V$, where $V$ is as in Proposition 6D, so Dehn's Lemma gives us a polyhedral disk $D_{1} \subset D_{0} \cup(U \cap V)$ with $\partial D_{1}=\partial D_{0}$. It is simple to check that $D_{1}$ meets the requirements.

If we apply Lemma $5 \mathrm{~B}$ to $D_{1}$, we obtain a disk $D^{\prime}$ in $E^{3}$ such that

(5.1) $\partial D^{\prime}=\partial D_{1}=\partial D$,

(5.2) $D^{\prime} \subset D_{1} \cup\left(\cup B_{i}\right) \subset D \cup U$,

(5.3) $D^{\prime} \subset$ int $S$.

$D^{\prime}$ satisfies conditions (i)-(iii) of Proposition 6A, and the proof is therefore complete.

Let $M$ be a 3-manifold, $S$ a 2-manifold in $M, V$ a component of $M \backslash S$, and $B$ a subset of $M$. A loop $f: \partial \Delta \rightarrow S$ can be shrunk to a point through $B \cap V$ if there is a homotopy $H_{t}: \partial \Delta \rightarrow M$ such that $H_{0}=f, H_{1}$ is constant, and $H_{t}(\partial \Delta) \subset B \cap V$ for all $t>0$. $S$ is 1-LC through $V$ at a point $p \in S$ if, for any neighborhood $B$ of $p$ in $M$, there is a neighborhood $B_{1}$ of $p$ in $B \cap S$ such that any loop $f: \partial \Delta \rightarrow B_{1}$ can be shrunk to a point through $B \cap V$.

COROllaRY 6A. Let $S$ be a 2-sphere in $E^{3}$ which is 1-LC through its interior at every point. Then $S$ is tame from the interior.

Proof. This follows from the observation: 
Proposition 6F. Let $B$ be a subset of $E^{3}$, and suppose that $i: \Delta \rightarrow S$ is an embedding such that $i(\partial \Delta)$ can be shrunk to a point through $B \cap$ int $S$. Then there is a blister $f$ of $i(\Delta)$ in int $S$ such that $f(\partial \Delta)=i(\partial \Delta)$ and $f(\Delta) \subset B$, and we have $O(F, f)$ $=i\left(\Delta^{\circ}\right)$, for any disk $F \subset S$ containing $i(\Delta)$.

Let $M$ be a 3-manifold, $S$ a 2-manifold in $M$, and $V$ a component of $M \backslash S$. A set $X$ in $S$ can be deformed into $V$ if there is a homotopy $H_{t}: X \rightarrow M$ such that $H_{0}=I$ and $H_{t}(X) \subset V$ for $t>0$.

Corollary 6B. Let $S$ be a 2-sphere in $E^{3}$ such that every Sierpinski curve in $S$ can be deformed into int $S$. Then $S$ is tame from the interior.

Proof. The following proposition is an adaptation of Theorem 14 in [6].

Proposition 6G. Let $E_{1}, E_{2}, \ldots, E_{k}, \ldots$ be a decreasing sequence of disks in $S$ whose intersection is a point $p \in \bigcap{E^{\circ}}_{k}$, and suppose that $p \cup\left(\cup \partial E_{k}\right)$ can be deformed into int $S$.

Then for any open set $B \subset E^{3}$ containing $E_{1}$, there is a blister $f$ of $E_{1}$ in int $S$ such that $p \in O\left(E_{1}, f\right) \subset(f)_{E_{1}} \subset B$ and $f(\partial \Delta)=\partial E_{K}$ for some $K$.

Proof. Let $H_{t}: p \cup\left(\cup \partial E_{k}\right) \rightarrow E^{3}$ be a homotopy such that $H_{0}=I$ and $H_{t}\left(p \cup\left(\bigcup \partial E_{k}\right)\right) \subset$ int $S$ for $t>0$. We may assume that $H_{t}\left(p \cup\left(\bigcup \partial E_{k}\right)\right) \subset B$ for each $t$; let $B^{\prime}$ be an open 3-cell in $B \backslash S$ containing $H_{1}(p) . H_{1}\left(\partial E_{K}\right)$ lies in $B^{\prime}$ for large enough $K$, and can be shrunk to a point in $B^{\prime}$. By Proposition $6 \mathrm{~F}$, there is then a blister $f$ of $E_{K}$ (and hence of $E_{1}$ ) in int $S$ such that $p \in E_{K}^{\circ}=O\left(E_{1}, f\right) \subset(f)_{E_{1}}$ $\subset B$ and $f(\partial \Delta)=\partial E_{K}$.

Proposition 6H. Let p be a point of $S, C$ a Cantor set in $S$. Then p is an inaccessible point of some Sierpinski curve in $S$ which misses $C$.

Proof. We just construct such a Sierpinski curve, using the fact that $C$ is 0-dimensional.

If $p$ is an inaccessible point of a Sierpinski curve $X$ in $S$, then there is a decreasing sequence of disks $E_{1}, E_{2}, \ldots, E_{k}, \ldots$ in $S$ such that $\partial E_{k} \subset X$ and $\cap E_{k}=\bigcap E_{k}^{\circ}=p$. Therefore, Propositions $6 \mathrm{G}$ and $6 \mathrm{H}$ together imply that $S$ satisfies condition (1) toward its interior at every point.

Remarks. (1) The hypothesis of Corollary 6B requires that any Sierpinski curve in $S$ can be continuously approximated from int $S$. For any 2-sphere $S$ in $E^{3}$, any Sierpinski curve $X$ in $S$, and any $\delta>0$, it follows from Bing's side approximation theorem [4, Theorem 16] that there is a $\delta$-homeomorphism $h: X \rightarrow \operatorname{int} S$.

(2) Theorem 6 is stated for 2-spheres in $E^{3}$, but its proof is based on a local criterion for tameness [8, Condition A], so Theorem 1 of [6] can be used with Lemma $5 \mathrm{~B}$ to extend our results to two-sided 2-manifolds in 3-manifolds.

\section{BIBLIOGRAPHY}

1. R. H. Bing, Approximating surfaces with polyhedral ones, Ann. of Math. 65 (1957), 456-483. 
2. R. H. Bing, An alternative proof that 3-manifolds can be triangulated, Ann. of Math. 69 (1959), 37-65.

3. - A surface is tame if its complement is 1-ULC, Trans. Amer. Math. Soc. 101 (1961), 294-305.

4. - Approximating surfaces from the side, Ann. of Math. (2) 77 (1963), 145-192.

5. - Pushing a 2-sphere into its complement, Michigan Math. J. 11 (1964), 33-45.

6. C. E. Burgess, Characterizations of tame surfaces in $E^{3}$, Trans. Amer. Math. Soc. 114 (1965), 80-97.

7. S. Eilenberg and N. E. Steenrod, Foundations of algebraic topology, Princeton Univ. Press, Princeton, N. J., 1952.

8. John Hempel, A surface in $S^{3}$ is tame if it can be deformed into each complementary domain, Trans. Amer. Math. Soc. 111 (1964), 273-287.

9. David W. Henderson, Extensions of Dehn's lemma and the loop theorem, Trans. Amer. Math. Soc. 120 (1965), 448-469.

10. C. D. Papakyriakopoulos, On Dehn's lemma and the asphericity of knots, Ann. of Math. 66 (1957), 1-26.

11. John Stallings, On the loop theorem, Ann. of Math. 72 (1960), 12-19.

12. Raymond Louis Wilder, Topology of manifolds, Amer. Math. Soc. Colloq. Publ. Vol. 32, Amer. Math. Soc. Providence, R. I., 1949.

\section{Arizona State University,}

Tempe, Arizona 